Article

\title{
Impacts of Temporal-Spatial Variant Background Ionosphere on Repeat-Track GEO D-InSAR System
}

\author{
Cheng $\mathrm{Hu}{ }^{1}$, Yuanhao $\mathrm{Li}^{1}{ }^{1}$ Xichao Dong ${ }^{1, *}$, Chang Cui ${ }^{1}$ and Teng Long ${ }^{1,2}$ \\ 1 School of Information and Electronics, Beijing Institute of Technology, Beijing 100081, China; \\ hucheng.bit@gmail.com (C.H.); 3120120331@bit.edu.cn (Y.L.); 1120121553@bit.edu.cn (C.C.); \\ longteng@bit.edu.cn (T.L.) \\ 2 Beijing Key Laboratory of Embedded Real-Time Information Processing Technology, Beijing 100081, China \\ * Correspondence: dongxichao@bit.edu.cn; Tel.: +86-10-6891-8127
}

Academic Editors: Zhong Lu, Xiaofeng Li and Prasad S. Thenkabail

Received: 25 July 2016; Accepted: 28 October 2016; Published: 4 November 2016

\begin{abstract}
An L band geosynchronous synthetic aperture radar (GEO SAR) differential interferometry system (D-InSAR) will be obviously impacted by the background ionosphere, which will give rise to relative image shifts and decorrelations of the SAR interferometry (InSAR) pair, and induce the interferometric phase screen errors in interferograms. However, the background ionosphere varies within the long integration time (hundreds to thousands of seconds) and the extensive imaging scene (1000 km levels) of GEO SAR. As a result, the conventional temporal-spatial invariant background ionosphere model (i.e., frozen model) used in Low Earth Orbit (LEO) SAR is no longer valid. To address the issue, we firstly construct a temporal-spatial background ionosphere variation model, and then theoretically analyze its impacts, including relative image shifts and the decorrelation of the GEO InSAR pair, and the interferometric phase screen errors, on the repeat-track GEO D-InSAR processing. The related impacts highly depend on the background ionosphere parameters (constant total electron content (TEC) component, and the temporal first-order and the temporal second-order derivatives of TEC with respect to the azimuth time), signal bandwidth, and integration time. Finally, the background ionosphere data at Isla Guadalupe Island $\left(29.02^{\circ} \mathrm{N}, 118.27^{\circ} \mathrm{W}\right)$ on $7-8$ October 2013 is employed for validating the aforementioned analysis. Under the selected background ionosphere dataset, the temporal-spatial background ionosphere variation can give rise to a relative azimuth shift of dozens of meters at most, and even the complete decorrelation in the InSAR pair. Moreover, the produced interferometric phase screen error corresponds to a deformation measurement error of more than $0.2 \mathrm{~m}$ at most, even in a not severely impacted area.
\end{abstract}

Keywords: Geosynchronous SAR (GEO SAR); temporal-spatial variation; background ionosphere; D-InSAR

\section{Introduction}

Geosynchronous synthetic aperture radar (GEO SAR) [1-3] runs at geosynchronous orbit which has the height of about $36,000 \mathrm{~km}$. It has a short revisit time of less than $24 \mathrm{~h}$ and extensive imaging coverage of more than $1000 \mathrm{~km}[4,5]$, which helps to realize the fast revisit and imaging of scenes of interest [6,7]. Therefore, the combination of GEO SAR and differential SAR interferometry (D-InSAR) can realize timely surface deformation detection, which has great advantages in the evaluation and forecast of natural disasters (earthquakes, landslides, volcano eruptions, etc.) [8-10].

The study of D-InSAR technology has a long history of more than 20 years. It can help to obtain the deformation information by virtue of accurate interferometric phases, which are deeply demonstrated in [11-15]. Nevertheless, the related study of D-InSAR processing in GEO SAR is rarely conducted. In 2002, Madsen et al. proposed the basic conception of using GEO D-InSAR to 
monitor the worldwide earthquake activities and analyzed the feasibility of the system [16]. Later, Monti-Guarneri and Bruno [2,17,18] studied the temporal advantages of a GEO D-InSAR system in surface deformation monitoring and the decorrelation impacts on GEO SAR data processing. Hu et al. [19] proposed methods of obtaining the optimal SAR interferometry (InSAR) pair for height and deformation retrieval in the repeat-track GEO InSAR system.

Although a GEO D-InSAR system has great potential in fast deformation detection, unfortunately, an L band GEO D-InSAR system will be seriously impacted by the ionosphere due to its low working frequency [20-22]. Meyer et al. [23] pointed out that the ionosphere could not only give rise to the relative image shift and the decorrelation of the InSAR pair, which will degrade the quality of the generated interferogram, but also the interferometric phase screen error could also be induced as a result of the degraded deformation retrieval accuracy. In Low Earth Orbit (LEO) SAR, because of the short integration time (less than $1 \mathrm{~s}$ ) and small imaging scene (about dozens of kilometers width), a significant amount of research about the ionosphere impacts on D-InSAR was conducted only based on the assumption of the ionosphere temporal-spatial frozen model, which considers the ionosphere to be invariant within the integration time and the scene [23,24]. However, GEO SAR has an integration time of more than $1 \mathrm{~min}$ (Figure 1a) and more than $1000 \mathrm{~km} \times 1000 \mathrm{~km}$ coverage, and the ionospheric total electron content (TEC) is obviously changing within the long integration time (Figure $1 \mathrm{~b}$ ) and the large spatial scope (Figure 1c). Therefore, the ionosphere temporal-spatial frozen model is invalid for the analysis of the impacts of background ionosphere variation on D-InSAR processing in a GEO SAR case. In addition, although some researches have applied the temporal-spatial background ionosphere variation model in previous studies, they mainly focus on the analysis of the impacts of the ionosphere on GEO SAR imaging $[1,25,26]$. Considering the above reasons, impacts of the temporal-spatial variant ionosphere on the deformation retrieval in GEO D-InSAR need to be specially considered. Generally, the ionospheric variation can be classified as two parts: the slow background ionosphere variation caused by the large-scale irregularities and the fast ionospheric scintillation caused by the small-scale irregularities $[1,27,28]$. Since occurrence of ionospheric scintillation has a special diurnal and geographical pattern (from early evening after sunset to midnight and at low and high latitude), ionospheric scintillation often occurs in a low probability. Even in the tropical zone, only $14 \%$ of the SAR images could be impacted by ionospheric scintillation [29]. More importantly, ionospheric scintillation could be avoided by applying the proper orbit optimization and the autofocusing algorithms [30,31]. Therefore, we focus on the impacts of the background ionosphere variation on GEO D-InSAR in this paper.

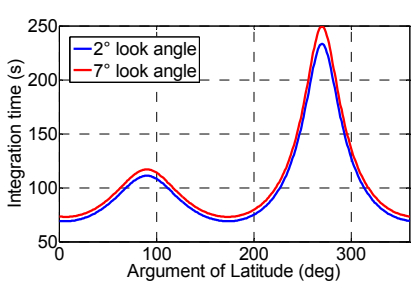

(a)

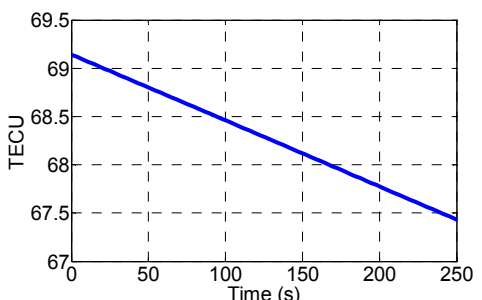

(b)

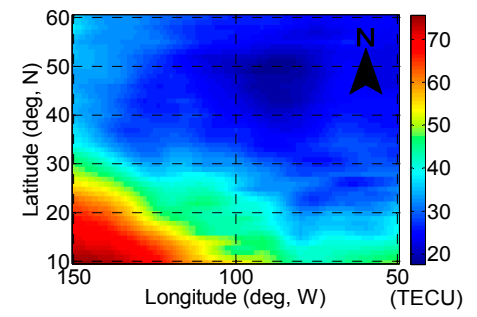

(c)

Figure 1. (a) Integration time of $\mathrm{L}$ band geosynchronous synthetic aperture radar (GEO SAR) in different orbit positions ( $0.24 \mathrm{~m}$ wavelength, $20 \mathrm{~m}$ azimuth resolution, a "figure- 8 " inclined orbit (0.07 eccentricity, $42,164 \mathrm{~km}$ semi-major axis and $53^{\circ}$ inclination)); (b) An example of the vertical TEC variation at the site $\left(10^{\circ} \mathrm{N}, 150^{\circ} \mathrm{W}\right)$ within $250 \mathrm{~s}$ (UTC Time 23:06:00-23:10:10, 16 September 2013); (c) An example of the vertical TEC variation within a spatial scope (UTC Time 23:00, 16 September 2013). The data in $(\mathbf{b}, \mathbf{c})$ derive from the United States Total Electron Content (USTEC) data released by the National Oceanic and Atmospheric Administration (NOAA) [32], which is near real-time assessment of the TEC by GPS signals. The USTEC uncertain in the vertical TEC (VTEC) is about 2 TECU (TECU is the unit of TEC, 1 TECU $=1 \times 10^{16}$ electrons $/ \mathrm{m}^{2}$ ) during quiet geomagnetic conditions. 
In this paper, aimed at the issue that the frozen background ionosphere model fails under the long integration time and large imaging scene of GEO SAR, we firstly deduce the GEO D-InSAR signal model in the presence of the temporal-spatial background ionosphere variation in Section 2. Then, based on the proposed model, expressions of the relative image shift and the decorrelation of the GEO InSAR pair, and the interferometric phase screen error model are given in Section 3. In addition, we illustrate the boundary parameters of the background ionosphere when these influences could be ignored in the D-InSAR processing. In Section 4, by utilizing the National Oceanic and Atmospheric Administration (NOAA) [32] TEC data at Isla Guadalupe Island, the relative image shift and the decorrelation in the interferometry processing brought by the temporal-spatial background ionosphere variation are simulated and analyzed. Moreover, the corresponding interferometric phase screen error is also evaluated and the results show that the obvious deformation measurement errors will be brought under the impacts of the temporal-spatial background ionosphere variation. Our studies are conducted based on the classical "figure- 8 " inclined orbit with an observation of America by an L band (0.24 m) GEO SAR system. Because of different orbits, required integration times and working frequencies, the performances of different GEO SAR systems under the impacts of the temporal-spatial background ionosphere variation are distinct. Finally, Section 5 concludes this paper.

\section{GEO D-InSAR Signal Model in the Presence of the Temporal-Spatial Background Ionosphere Variation}

The sketch map of a GEO D-InSAR system in the presence of the background ionosphere is shown in Figure 2. Point $\mathbf{P}$ is the target on the ground. $R_{G}$ is the slant range of GEO SAR. $L_{G}$ is the synthetic aperture of GEO SAR, and $l_{P}$ is the equivalent projections of the corresponding synthetic apertures on the ionosphere. $H_{\text {iono }}$ is the representative height of the ionosphere. The color variation of the ionosphere represents the TEC variation. Because of the long integration time of GEO SAR, TEC is obviously changing, not only between the revisiting interval, but within the integration time.

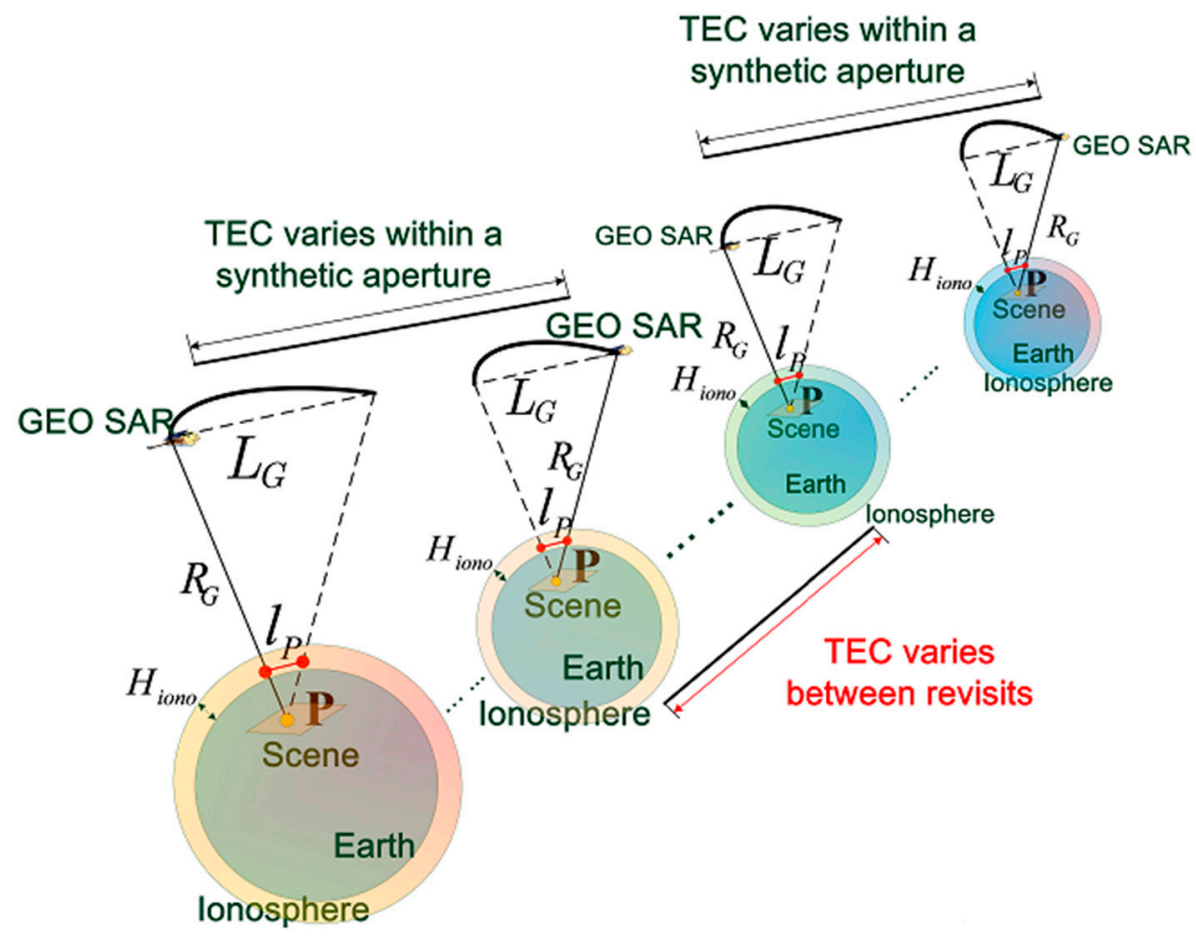

Figure 2. Sketch map of a GEO D-InSAR system in the presence of the background ionosphere. 
Considering that the impacts of the background ionosphere variation on GEO SAR signals vary along with the slow time and the spatial position, the TEC variation in one track of GEO SAR, based on the series expansion with respect to the slow time, is written as:

$$
\operatorname{TEC}\left(t_{a} ; \mathbf{P}\right)=\operatorname{TEC}_{0}(\mathbf{P})+k_{1}(\mathbf{P}) t_{a}+k_{2}(\mathbf{P}) t_{a}^{2}+\ldots
$$

where $\mathbf{P}$ is the position vector of a target in the scene, $t_{a}$ is the azimuth time, $T E C\left(t_{a} ; \mathbf{P}\right)$ is the TEC of point $\mathbf{P}$ changing with the slow time along the signal propagation path (STEC), and $T E C_{0}$ is the constant component of $T E C\left(t_{a} ; \mathbf{P}\right)$, which corresponds to the TEC value in the aperture center moments. $k_{i}(\mathbf{P})(i=1,2, \ldots)$ is the temporal $i$ th-order derivative of TEC with respect to the azimuth time.

Generally, under a design of moderate resolution $(20 \mathrm{~m})$, the ionospheric pierce trajectory of the GEO SAR with a "figure-8" nadir-point trajectory is less than $3 \mathrm{~km}$ (smaller than that in the LEO SAR case (5-10 km)). The TEC variation caused by the spatial ionospheric pierce points (IPPs) variation (about $10^{-2} \sim 10^{-1} \mathrm{TECU}$ ) is far smaller than the TEC variation of a spatial invariant IPP within the integration time of GEO SAR (about $10^{-1} \sim 1$ TECU). Therefore, we ignore the impacts of TEC variation caused by the spatial IPPs variation. Based on Equation (1), the GEO SAR signal $s\left(f_{a}, t_{a} ; \mathbf{P}\right)$ is given as (in the range frequency domain and the azimuth time domain):

$$
s\left(f_{a}, t_{a} ; \mathbf{P}\right)=W\left(f_{a}, t_{a} ; \mathbf{P}\right) \cdot \exp \left[j(\frac{\pi f_{a}^{2}}{k_{r}}-\underbrace{\frac{4 \pi K \cdot T E C\left(t_{a} ; \mathbf{P}\right)}{c f_{a}}}_{\phi_{i}\left(f_{a}\right)}-4 \pi \frac{f_{0} R\left(t_{a} ; \mathbf{P}\right)}{c})\right]
$$

where $k_{r}$ is the frequency modulation rate, $f_{a}$ is the range frequency, $f_{0}$ is the carrier center frequency, $c$ is light velocity, $R\left(t_{a} ; \mathbf{P}\right)$ is the accurate slant range, $K=40.28 \mathrm{~m}^{3} / \mathrm{s}^{2}$ is a constant, $W\left(f_{a}, t_{a} ; \mathbf{P}\right)$ is the signal envelope, and $\phi_{i}\left(f_{a}\right)$ represents the phase induced by the background ionospheric impacts.

Considering that the designed frequency bandwidth of GEO SAR is about $20 \mathrm{MHz}$ under the moderate resolution of $20 \mathrm{~m}$ and the variation of TEC is generally smaller than 100 TECU in natural conditions, dispersion in range could be ignored [25]. Thus, after imaging processing in the time domain, we have

$$
s\left(t-\tau, t_{a}, \mathbf{P}\right)=\bar{W}\left(t-\tau, t_{a} ; \mathbf{P}\right) \cdot \exp \left[-j\left(4 \pi \frac{f_{0} R_{c}(\mathbf{P})}{c}+\phi_{i o n}(\mathbf{P})\right)\right]
$$

where $t$ is the fast time, $\tau$ is the imaged position of the target in range, $\bar{W}\left(t-\tau, t_{a} ; \mathbf{P}\right)$ is the envelope of the signal, $R_{\mathcal{c}}(\mathbf{P})$ is the slant from the target to the aperture center, and $\phi_{i o n}(\mathbf{P})$ is the integrated phase error brought by the background ionosphere variation after imaging, which is expressed as

$$
\phi_{\text {ion }}(\mathbf{P})=\arg \left\{\int_{-T_{a} / 2}^{T_{a} / 2} \exp \left[-j \frac{4 \pi K \cdot T E C\left(t_{a} ; \mathbf{P}\right)}{c f_{0}}\right] d t_{a}\right\}
$$

where $T_{a}$ represents the integration time.

Considering the D-InSAR processing, after complex multiplication of the GEO InSAR pair, the interferometric signal is expressed as

$$
s_{M}\left(t-\tau, t_{a, T_{0}} ; \mathbf{P}\right) s_{S}^{*}\left(t-\tau, t_{a, T_{0}+n T_{r}} ; \mathbf{P}\right)=\widetilde{W}\left(t-\tau, t_{a} ; \mathbf{P}\right) \exp \left[-j\left(4 \pi \frac{f_{0} \Delta R_{\mathcal{c}}(\mathbf{P})}{c}+\Delta \phi_{\text {ion }}(\mathbf{P})\right)\right]
$$

where $s_{M}$ and $s_{S}$ are the master image and the slave image of the InSAR pair, respectively. $\widetilde{W}\left(t-\tau, t_{a} ; \mathbf{P}\right)$ is the envelope of the interferometric signal, $\Delta R_{c}(\mathbf{P})$ is the slant difference from the target to the aperture center. $T_{0}$ is the aperture center moments of the first track and $T_{r}$ is the revisit 
period. $n$ is an integer to represent the number of revisit periods which have been passed. $\Delta \phi_{\text {ion }}(\mathbf{P})$ is the interferometric phase brought by the background ionosphere variation, which is shown as:

$$
\Delta \phi_{\text {ion }}(\mathbf{P})=\arg \left\{\int_{-T_{a} / 2}^{T_{a} / 2} \exp \left[-j \frac{4 \pi K \cdot T E C_{M}\left(t_{a} ; \mathbf{P}\right)}{c f_{0}}\right] d t_{a}\right\}-\arg \left\{\int_{-T_{a} / 2}^{T_{a} / 2} \exp \left[-j \frac{4 \pi K \cdot T E C_{S}\left(t_{a} ; \mathbf{P}\right)}{c f_{0}}\right] d t_{a}\right\}
$$

where $T E C_{M}\left(t_{a} ; \mathbf{P}\right)$ and $T E C_{S}\left(t_{a} ; \mathbf{P}\right)$ are the TECs of point $\mathbf{P}$, changing with the slow time during two acquisitions of the GEO InSAR pair.

\section{Impacts of the Temporal-Spatial Background Ionosphere Variation on GEO D-InSAR}

\subsection{Relative Image Shift and the Decorrelation of the InSAR Pair}

The signal envelope delay brought by the temporal-spatial background ionosphere variation during two acquisitions of the GEO InSAR pair will give rise to a relative range shift of the InSAR pair $\Delta R$. The range shift of each image in the InSAR pair is determined by the first-order derivative of $\phi_{i}\left(f_{a}\right)$ in Equation (2) with respect to $f_{a}$ at $f_{0}$. Thus, $\Delta R$ is shown as:

$$
\Delta R(\mathbf{P}) \approx \frac{K}{f_{0}^{2}}\left\{T E C_{0, M}(\mathbf{P})-T E C_{0, S}(\mathbf{P})\right\}
$$

where $T E C_{0, M}(\mathbf{P})$ and $T E C_{0, S}(\mathbf{P})$ are the constant components of $T E C_{M}\left(t_{a} ; \mathbf{P}\right)$ and $\operatorname{TEC}_{S}\left(t_{a} ; \mathbf{P}\right)$, respectively.

As the azimuth shift of each SAR image is proportional to the temporal first-order derivative of TEC with respect to the azimuth time [25], the relative azimuth shift of the InSAR pair $\triangle \mathrm{a}$ can be expressed as:

$$
\Delta \mathrm{a}(\mathbf{P}) \approx \frac{2 K}{c f_{0}}\left\{\frac{v_{M, 0} k_{1, M}(\mathbf{P})}{f_{d r 0, M}}-\frac{v_{S, 0} k_{1, S}(\mathbf{P})}{f_{d r 0, S}}\right\}
$$

where $v_{M, 0}$ and $v_{S, 0}$ are the velocities of the two tracks of the InSAR pair at the aperture center moments, respectively. $f_{d r 0, M}$ and $f_{d r 0, S}$ are the Doppler frequency modulation rates of the two tracks of the InSAR pair at the aperture center moments, respectively. $k_{1, M}$ and $k_{1, S}$ are the temporal first-order derivatives of TECs during two acquisitions of the GEO InSAR pair, respectively.

According to Equations (7) and (8), the constant TEC components during two acquisitions of the GEO InSAR pair and the carrier center frequency determine the relative range shift, while the relative azimuth shift depends on the temporal first-order derivatives of TECs during two acquisitions of the GEO InSAR pair, the velocities of the two tracks of the InSAR pair at the aperture center moments and the Doppler frequency modulation rates of the two tracks of the InSAR pair at the aperture center moments. Generally, the relative range shift and the azimuth shift will give rise to the mismatch of the InSAR pair, and finally cause the decorrelation, which is shown as:

$$
\gamma_{i}=\gamma_{1} \gamma_{2}
$$

where $\gamma_{i}$ is the coefficient that corresponds to the mismatch decorrelation,

$$
\gamma_{1}=\operatorname{sinc}\left\{\frac{2 K B \sin \theta_{r}}{c f_{0}^{2}}\left[T E C_{0, M}(\mathbf{P})-T E C_{0, S}(\mathbf{P})\right]\right\}
$$

where $\gamma_{1}$ represents the mismatch decorrelation in range, $\theta_{r}$ is the incident angle, $B$ is the bandwidth,

$$
\gamma_{2}=\operatorname{sinc}\left\{\frac{2 K T_{a}}{c f_{0}}\left(k_{1, M}(\mathbf{P})-k_{1, S}(\mathbf{P})\right)\right\}
$$

where $\gamma_{2}$ represents the mismatch decorrelation in azimuth. 
On one hand, large differences in the constant TEC components and the temporal first-order derivatives of TECS during two acquisitions of the GEO InSAR pair will aggravate the decorrelation. On the other hand, a larger bandwidth and incident angle (higher range resolution), and a longer integration time (higher azimuth resolution) can also make the coherence decrease. In addition, as the constant components and the temporal first-order derivatives of TECS are spatial-varying, the produced relative shifts and the decorrelation of the InSAR pair are not uniform in the scene. To obtain a high coherence in the generated interferogram, we need co-registration to eliminate the relative shifts of the InSAR pair. Nevertheless, the regularity of the relative shifts of the InSAR pair will decrease when the constant components and the temporal first-order derivatives of TECS change dramatically within the scene. A high-order polynomial model could be utilized under the condition to realize a more accurate co-registration. Likewise, we can ignore the relative shifts of the InSAR pair when the differences between the constant components and the temporal first-order derivatives of TECs during two acquisitions are small. Generally, the accuracy requirement for co-registration is the mismatch of the pixels that should be smaller than $1 / 10$ of a pixel [12], which can only bring a decorrelation of less than $4 \%$. Hereby, without considering the changes of the velocities and the Doppler frequency modulation rates of the two tracks, the boundary parameters of the background ionosphere, when the impacts of the relative image shift and the decorrelation of the GEO SAR interferometry pair could be ignored, can be expressed as:

$$
\left\{\begin{array}{l}
\left|\Delta T E C_{0}(\mathbf{P})\right| \leq \frac{c f_{0}^{2}}{20 B \sin \theta_{r} K} \\
\left|\Delta k_{1}\right| \leq \frac{c f_{0}}{20 T_{a} K}
\end{array} .\right.
$$

\subsection{Interferometric Phase Screen Error}

Based on the previous signal model, $\Delta \phi_{\text {ion }}$ will introduce errors in the interferometric phase screen, resulting in deformation errors. Thus, it is necessary to discuss its impacts. Combining Equation (1) in Equation (4), we obtained:

$$
\phi_{\text {ion }}(\mathbf{P})=\arg \left\{\int_{-T_{a} / 2}^{T_{a} / 2} \exp \left[-j \frac{4 \pi K}{c f_{0}} \cdot\left(\operatorname{TEC}_{0}(\mathbf{P})+k_{1}(\mathbf{P}) t_{a}+k_{2}(\mathbf{P}) t_{a}^{2}+\ldots\right)\right] d t_{a}\right\} .
$$

Generally, impacts of the temporal third-order derivative of TEC with respect to the azimuth time are about $10^{-8}-10^{-10}$ TECU and it is smaller than 0.01 TECU after the integration within the integration time. Thus, we ignore the impacts of the TEC variation components above the temporal second-order derivative. By deduction (See Appendix A for details), the temporal-spatial variation interferometric phase screen error $\Delta \phi_{\text {ion }}$ can be written as

$$
\Delta \phi_{\text {ion }}(\mathbf{P})=\frac{4 \pi K}{c f_{0}}\{\underbrace{\widehat{\Phi}_{0}(\mathbf{P})}_{\text {space Variation }}-\underbrace{\widehat{\Phi}_{1}(\mathbf{P})+\widehat{\Phi}_{2}(\mathbf{P})}_{\text {Time-spatial Variation }}\}
$$

where $\widehat{\Phi}_{0}(\mathbf{P})$ is the component of spatial background ionosphere variation, $\widehat{\Phi}_{1}(\mathbf{P})$ and $\widehat{\Phi}_{2}(\mathbf{P})$ are the components of temporal-spatial background ionosphere variation, which are shown as:

$$
\left\{\begin{aligned}
\widehat{\Phi}_{0}(\mathbf{P}) & =T E C_{0, M}(\mathbf{P})-T E C_{0, S}(\mathbf{P}) \\
\widehat{\Phi}_{1}(\mathbf{P}) & =\left(\frac{k_{1, M}(\mathbf{P})^{2}}{4 k_{2, M}(\mathbf{P})}-\frac{k_{1, S}(\mathbf{P})^{2}}{4 k_{2, S}(\mathbf{P})}\right) \\
\widehat{\Phi}_{2}(\mathbf{P}) & =\frac{c f_{0}}{4 \pi K}\left(\arctan \left[\frac{4 \pi K}{3 c f_{0}}\left(\frac{3 k_{1, M}(\mathbf{P})^{2}}{4 k_{2, M}(\mathbf{P})}+\frac{T_{a}^{2} k_{2, M}(\mathbf{P})}{4}\right)\right]\right. \\
& \left.-\arctan \left[\frac{4 \pi K}{3 c f_{0}}\left(\frac{3 k_{1, S}(\mathbf{P})^{2}}{4 k_{2, S}(\mathbf{P})}+\frac{T_{a}^{2} k_{2, S}(\mathbf{P})}{4}\right)\right]\right)
\end{aligned}\right.
$$


where $k_{2, M}$ and $k_{2, S}$ are the temporal second-order derivative of TECs during two acquisitions of the GEO InSAR pair, respectively.

According to Equation (14), it not only contains the component of spatial background ionosphere variation, but it includes the components of the temporal-spatial background ionosphere variation. $\widehat{\Phi}_{0}(\mathbf{P})$ only relates to the constant components of TECs during two acquisitions of the GEO InSAR pair, while $\widehat{\Phi}_{1}(\mathbf{P})$ and $\widehat{\Phi}_{2}(\mathbf{P})$ highly depend on the temporal derivatives of TECs and the integration time. When $k_{2, M}$ and $k_{2, S}$ are small, as the impacts of the $k_{1, M}$ and $k_{1, S}$ are symmetrical within the whole aperture, the phase error after integration is dominated by $\widehat{\Phi}_{0}(\mathbf{P})$. In contrast, when $k_{2, M}$ and $k_{2, S}$ are large, only Equation (14) could be utilized to describe the interferometric phase screen error brought by the temporal-spatial background ionosphere variation accurately.

$\Delta \phi_{\text {ion }}(\mathbf{P})$ will disturb the correct interferometric phase and introduce the deformation detection error $\Delta d_{i o n}$, which is given as:

$$
\Delta d_{i o n}(\mathbf{P})=-\frac{c}{4 \pi f_{0}} \cdot \Delta \phi_{i o n}(\mathbf{P})
$$

\section{Simulation and Discussion}

\subsection{Experiment Data and the Preprocessing}

To verify the theoretical analysis, the USTEC data released by the NOAA is utilized. USTEC data has a temporal sampling rate of $15 \mathrm{~min}$ and the spatial sampling interval of $1^{\circ}$. The constant component, the temporal first-order derivative component and the temporal second-order derivative component of $T E C$, with respect to the azimuth time, are obtained by a second or higher order polynomial fitting of the multi-temporal sampling TEC data.

Shown in Figure 3a, the inclined GEO SAR orbit with a "figure-8" nadir-point trajectory is utilized. Its orbit parameters are given in Table 1. Our scene of interest in the simulation is an area of more than $1000 \mathrm{~km} \times 1000 \mathrm{~km}$, which has a center at Isla Guadalupe Island $\left(29.02^{\circ} \mathrm{N}, 118.27^{\circ} \mathrm{W}\right)$ in the Pacific Ocean. As shown in the sketch map in Figure 3b, the GEO SAR signal obliquely traversed in the space to illuminate target A. Thus, the calculated IPPs of target A should be located at point B. Meanwhile, the VTEC should be transformed into STEC as well. Moreover, as discussed in Section 2, since the TEC variation caused by the spatial IPPs variation is negligible, we ignore the impacts of $T E C$ variation caused by the spatial IPPs variation in the following data processing.

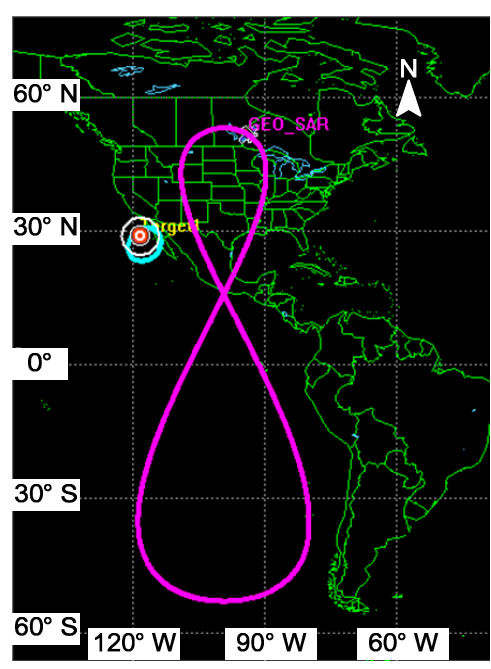

(a)

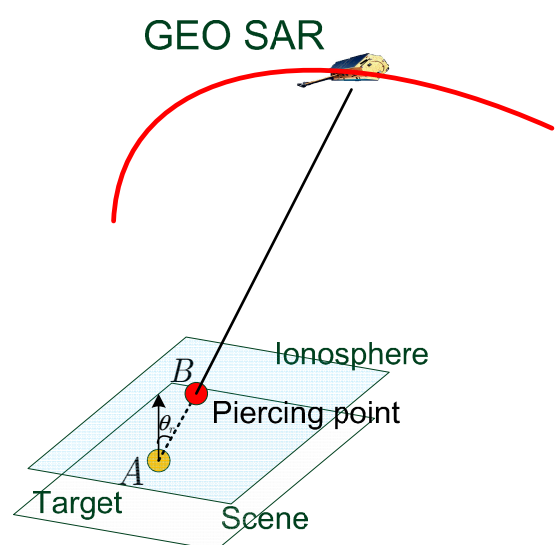

(b)

Figure 3. (a) GEO SAR "figure-8" nadir-point trajectory; (b) Sketch map of the oblique path of the GEO SAR signal in space. 
Table 1. GEO SAR orbit parameters.

\begin{tabular}{cccc}
\hline Items & Value & Items & Value \\
\hline Semi-major axis $(\mathrm{km})$ & $42,164.170$ & Wavelength $(\mathrm{m})$ & 0.24 \\
$\begin{array}{c}\text { Inclination (degrees) } \\
\text { Argument of Perigee }\end{array}$ & 53 & Eccentricity & 0.07 \\
(degrees) & 270 & Right Ascension of Ascending & 210 \\
\hline
\end{tabular}

The GEO SAR system has a signal bandwidth of $80 \mathrm{MHz}$ and an integration time of $250 \mathrm{~s}$. The SAR data are obtained at orbit positions near apogee. Considering the short revisit time of the GEO SAR system, we select the temporal baseline of 1 day in the following simulation. The interferometric baseline is about $1.3 \mathrm{~km}$. The TEC data used in the InSAR pair were acquired at UTC 22:00-23:00, 7 October 2013 and UTC 22:00-23:00, 8 October 2013, respectively. The acquisition time interval corresponds to the local time interval from noon to the sunset, which makes the utilized TEC data characterized by larger absolute TEC values and obvious TEC variation, and helps to study the impacts of temporal-spatial background ionosphere variation on GEO D-InSAR. After the IPPs calculations and the transformation from the VTEC to the STEC, based on the selected orbit, the TEC data during two acquisitions of the InSAR pair is shown in Figure $4\left(0.1^{\circ}\right.$ spatial interval after triangle-based linear interpolation processing).

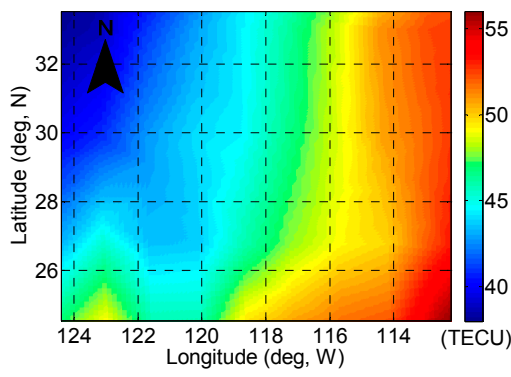

(a)

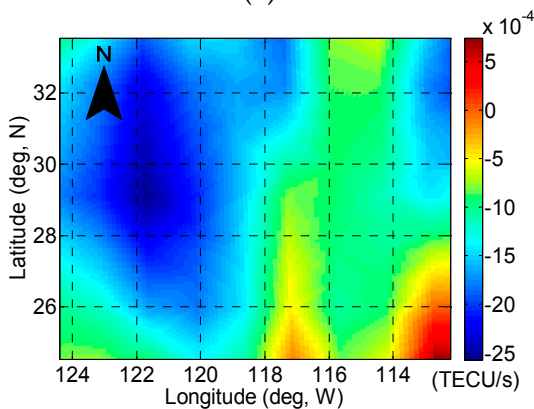

(c)

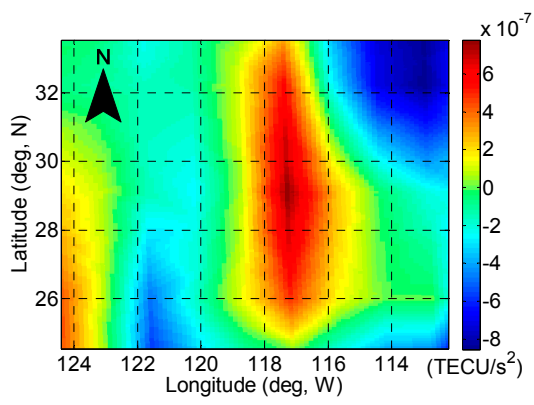

(e)

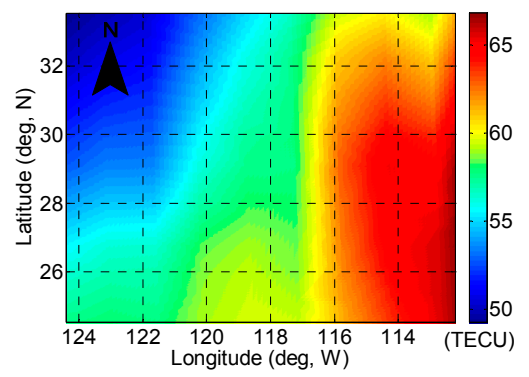

(b)

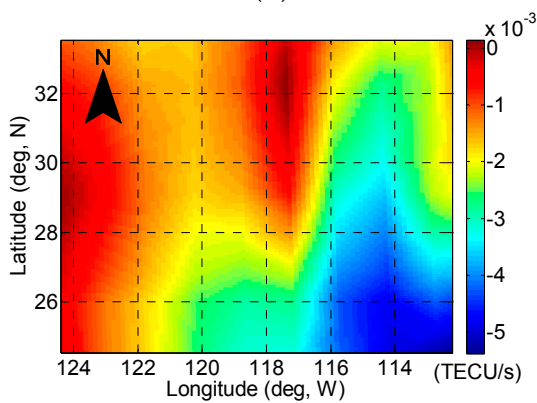

(d)

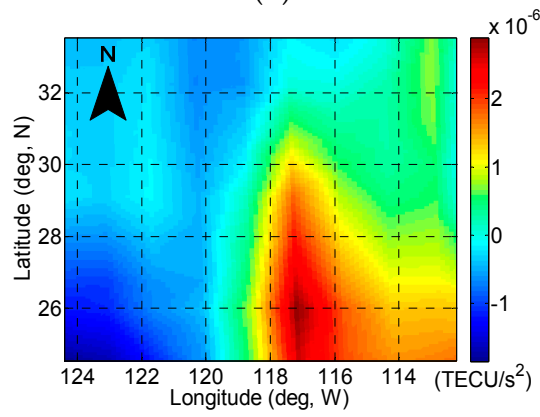

(f)

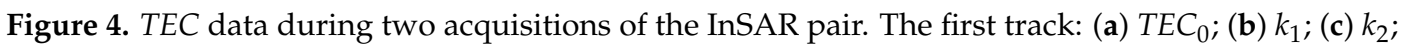
the second track: (d) $T E C_{0} ;(\mathbf{e}) k_{1} ;(\mathbf{f}) k_{2}$. 


\subsection{Impacts of the Relative Image Shift and the Decorrelation of the InSAR Pair}

Based on the TEC data (as shown in Figure 4) and the GEO SAR configuration, the relative image shift and the decorrelation of the InSAR pair are obtained and shown in Figure 5. It can be concluded that the largest range relative shift is less than $4 \mathrm{~m}$, which gives rise to a loss of correlation coefficient of no more than 0.13. As the difference of the constant components of the TECS during the InSAR pair acquisitions are only about $10 \mathrm{TECU}$, the corresponding range relative shift is limited. The obvious range relative shift will only be generated in the case of a large difference in the constant components of the TECS during the InSAR pair acquisitions. For instance, in a condition of a 50 TECU difference for the constant components of the TECS during the InSAR pair acquisitions, the generated range relative shift is larger than $13 \mathrm{~m}$ and a total decorrelation will occur. Nevertheless, it is generally uncharacteristic that there will be a large bias between the TEC data during the two acquisitions of the InSAR pair in natural conditions.

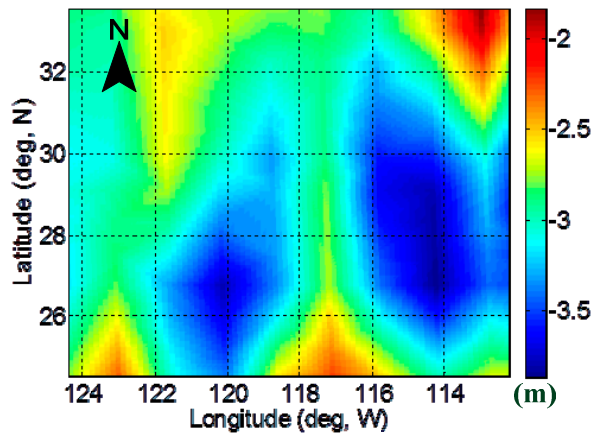

(a)

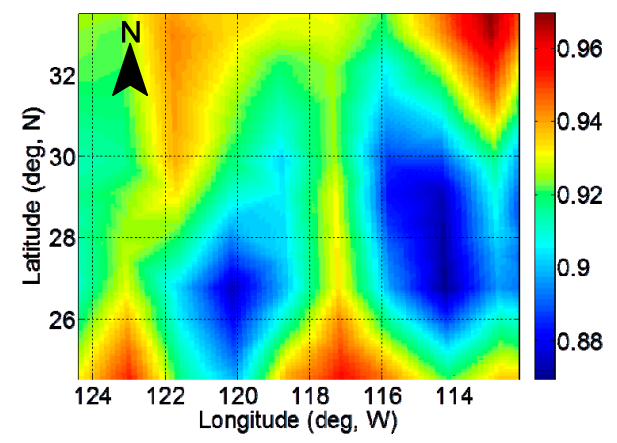

(c)

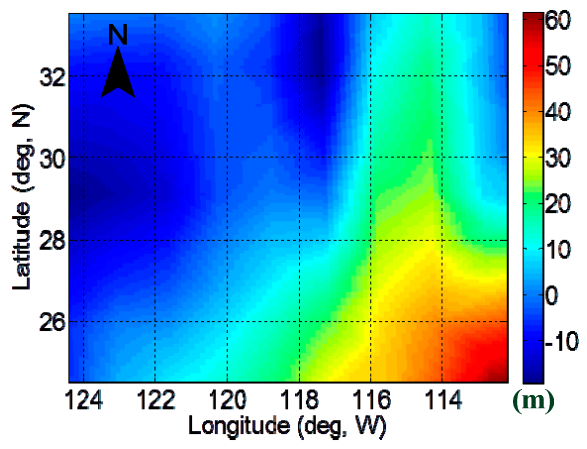

(b)

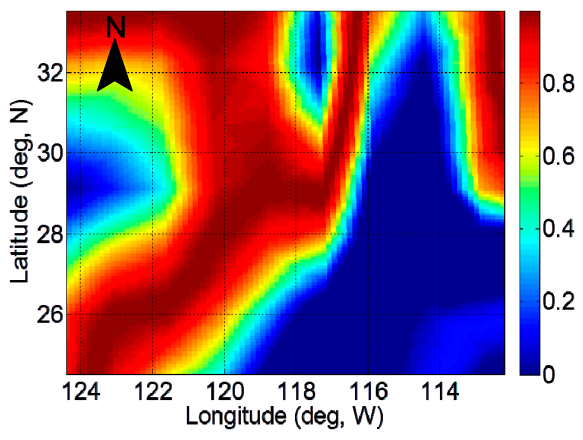

(d)

Figure 5. Impacts of the relative image shift and the decorrelation of the InSAR pair. (a) Range relative shift; (b) Azimuth relative shift; (c) Correlation coefficient only under the impacts of the range relative shift; (d) Correlation coefficient only under the impacts of the azimuth relative shift.

In contrast, the azimuth relative shift and the corresponding decorrelation are obvious under the selected temporal first-order derivatives of TECs during two acquisitions of the GEO InSAR pair and the integration time. There are many parts with the azimuth relative shifts of more than $20 \mathrm{~m}$ in Figure 5b, which result in a total decorrelation in the interferogram. Only less than half of the interferogram keeps the correlation coefficients of more than 0.6.

Based on Equation (12) and the parameters in Table 1, the boundary parameters of the background ionosphere, when the impacts of the relative image shift and the decorrelation of the GEO InSAR pair can be ignored at apogee, is given in Table 2. It could be concluded that the impacts are negligible when the resolution of the SAR image is coarse and the variation of the parameters of the TECS between the two acquisitions of the GEO InSAR pair is limited. Otherwise, a high accurate co-registration is needed in the processing. 
Table 2. Boundary parameters of the background ionosphere when the impacts of the relative image shift and the decorrelation of the GEO InSAR pair could be ignored under different resolutions (apogee).

\begin{tabular}{|c|c|c|}
\hline Resolution (m) & $\left|\Delta T E C_{0}\right|$ (Total Electron Content Unit (TECU)) & $\left|\Delta k_{1}\right|(\mathrm{TECU} / \mathrm{s})$ \\
\hline 100 & $<38.8$ & $<9.65 \times 10^{-4}$ \\
\hline 20 & $<7.8$ & $<1.93 \times 10^{-4}$ \\
\hline 5 & $<1.9$ & $<4.83 \times 10^{-5}$ \\
\hline
\end{tabular}

\subsection{Interferometric Phase Screen Error}

Based on Equation (14), the relationships between the integration time and the interferometric phase under the different cases of the constant components, and the temporal first-order and the second-order derivatives of TEC with respect to the azimuth time, are shown in Figure 6. Firstly, under a specified TEC condition, the interferometric phase brought by the impacts of the temporal-spatial background ionosphere variation on GEO SAR increases when the integration time becomes longer. In additional, the tendencies of variations of the blue line and the red line with respect to the integration time in the figure are the same. Only the initial interferometric phase is different. Thus, the difference in the constant components of the TECS only gives rise to a fixed bias in the interferometric phase. In contrast, the variations of $\Delta k_{1}$ and $\Delta k_{2}$ have obvious impacts on the interferometric phase. Based on the changing tendencies of the blue line, the light blue line and the green line, it could be concluded that the interferometric phase decreases with the increase of $\Delta k_{1}$ under a specified integration time and $\Delta k_{2}$. According to Equation (15), when $\Delta k_{1}$ is small, the product between $\Delta k_{2}$ and the integration time approximately dominates the phase error. Conversely, a larger $\Delta k_{1}$ will decrease the effect of the product between $\Delta k_{2}$ and the integration time on the phase error. As a result, the interferometric phase becomes insensitive to the variation of the integration time for a larger $\Delta k_{1}$. As for $\Delta k_{2}$, when $\Delta k_{2}$ is increasing, the interferometric phase becomes larger under a specified integration time and $\Delta k_{1}$ (the blue line, the yellow line and the purplish red line). In this case, the interferometric phase becomes more sensitive to the variation of the integration time.

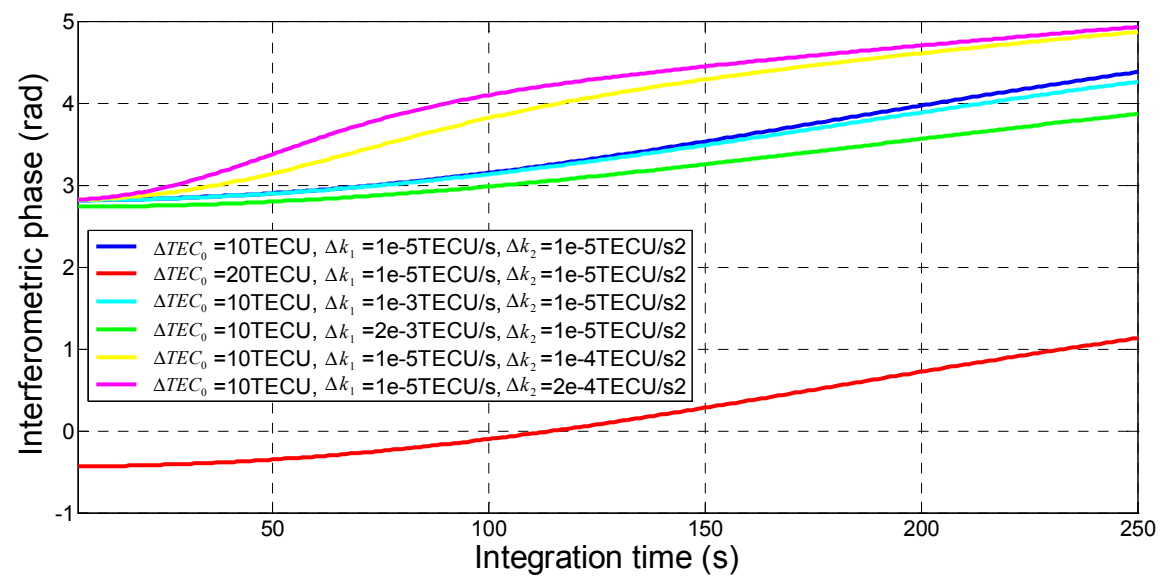

Figure 6. Relationships between the integration time and the interferometric phase under the different cases of the constant components, and the temporal first-order and the temporal second-order derivatives of TEC with respect to the azimuth time (TEC parameters in the first track: $T E C_{0}=10 \mathrm{TECU}$, $k_{1}=1 \times 10^{-5} \mathrm{TECU} / \mathrm{s}, k_{2}=1 \times 10^{-5} \mathrm{TECU} / \mathrm{s}^{2}, \triangle T E C_{0}$ is the differences of the constant components of TECs during two acquisitions of the GEO InSAR pair, $\Delta k_{1}$ and $\Delta k_{2}$ are the differences of the temporal first-order and the temporal second-order derivatives of TECs during two acquisitions of the GEO InSAR pair, respectively). 
Utilizing the USTEC data in Figure 4 and Equation (14), the interferometric phase screen errors generated by $\widehat{\Phi}_{1}(\mathbf{P})$ and $\widehat{\Phi}_{2}(\mathbf{P})$ are shown in Figure 7a. The spatial distributed fringe frequency is brought by the interferometric phase screen ranges from less than one circle to multiple circles per one million square kilometers. Thus, the interferometric phase screen errors could give rise to deformation errors of up to several meters in the areas with dense fringes. We select the interferometric phase screen in the area marked by the white rectangle of Figure $7 \mathrm{a}$, which has a relatively lower fringe frequency for the detailed deformation error analysis. The corresponding deformation retrieval error is given in Figure $7 \mathrm{~b}$. Even in the area with a lower fringe frequency, the largest deformation retrieval error in the scene is higher than $0.2 \mathrm{~m}$ and the mean square root error of the deformation is $0.13 \mathrm{~m}$, which cannot satisfy the requirements for the deformation retrieval accuracy in any engineering applications. Therefore, some compensation algorithms based on Persistent Scatterer technology (PS) [4,33-37] or some similar methods based on the high quality coherent points are really needed to eliminate the serious impacts of the interferometric phase screen errors brought by the temporal-spatial background ionosphere variation in GEO D-InSAR processing in the future.

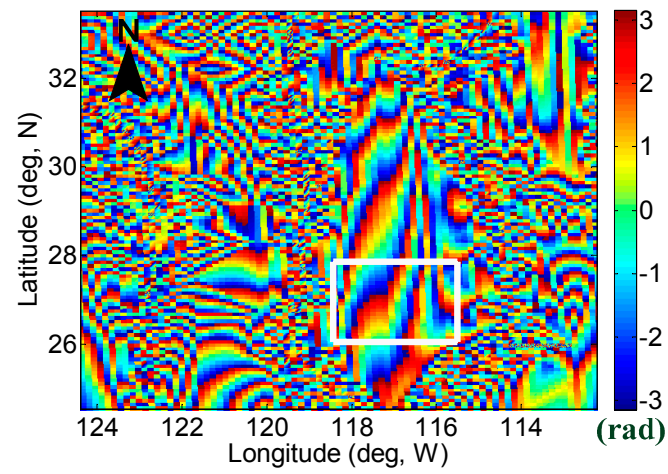

(a)

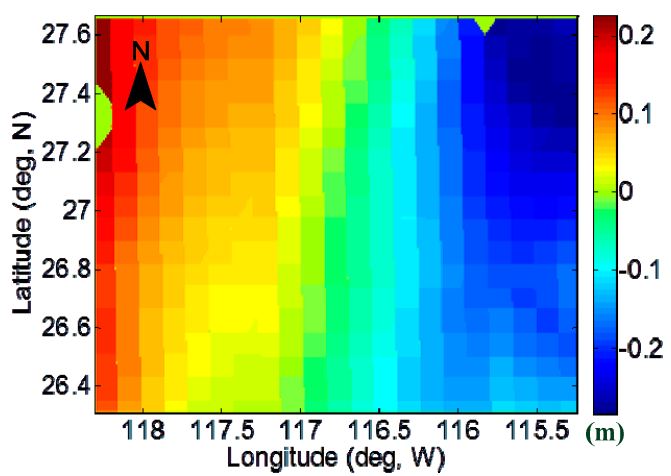

(b)

Figure 7. (a) Interferometric phase screen error generated by $\widehat{\Phi}_{1}(\mathbf{P})$ and $\widehat{\Phi}_{2}(\mathbf{P})$; (b) Deformation retrieval error in the white rectangle area.

\section{Conclusions}

Based on the temporal-spatial background ionosphere variation model, this paper focused on the analysis of impacts of the temporal-spatial background ionosphere variation on GEO D-InSAR processing, including the relative image shift and the decorrelation of the GEO InSAR pair, and the interferometric phase screen errors. It addresses the issue that the conventional frozen background ionosphere model used in LEO SAR is no longer valid for GEO SAR because of its long integration time and extensive imaging scene. The quantitative analysis is conducted by simulations based on the USTEC data. We draw two meaningful conclusions from our research.

Firstly, the differences of the constant TEC components and the temporal first-order derivatives of TEC with respect to the azimuth time during two acquisitions of the GEO InSAR pair, signal bandwidth and the integration time will give rise to an obvious relative image shift and the decorrelation of the GEO InSAR pair. Under the condition of GEO SAR parameters and the selected TEC data in our paper, a serious relative image shift in azimuth (dozens of meters) and the decorrelation of the GEO InSAR pair (almost total decorrelation) occur. When the parameters of the TEC variation during the acquisition of the GEO InSAR pair are small, the impacts of the relative image shift and the decorrelation of the GEO SAR interferometry pair could be ignored. For instance, the requirement of the TEC parameters are $\left|\triangle T E C_{0}\right|<7.8 \mathrm{TECU}$ and $\left|\Delta k_{1}\right|<1.93 \times 10^{-4} \mathrm{TECU} / \mathrm{s}$ under the moderate resolution of $20 \mathrm{~m}$.

Secondly, the temporal-spatial part of the interferometric phase screen error $\widehat{\Phi}_{1}(\mathbf{P})$ and $\widehat{\Phi}_{2}(\mathbf{P})$ highly depend on the temporal derivatives of TECs and the integration time. During two acquisitions of the GEO InSAR pair, a small variation of the temporal first-order derivatives of TEC with respect to 
the azimuth time and a larger variation of the temporal second-order derivatives of TEC with respect to the azimuth time, will give rise to the obvious temporal-spatial part of the interferometric phase screen error. Under the selected TEC parameters, the temporal-spatial part of the interferometric phase screen error will cause a deformation retrieval error of more than $0.2 \mathrm{~m}$, even in the area with relatively small impacts of the ionosphere. Some compensation algorithms based on PS technology could have great potential in suppressing the deformation retrieval error, which will be our study focus in the future.

Acknowledgments: This work is supported by National Natural Science Foundation of China (Grant No. 61225005, 61471038, 61501032, 61427802, 61120106004), Beijing Natural Science Foundation (Grant No. 4162052) and Chang Jiang Scholars Program (T2012122) and 111 project of China under Grant B14010.

Author Contributions: Y.L. and C.H. conceived and designed the methods; X.D. and Y.L. performed the simulation; C.C. and T.L. analyzed the data; X.D. and Y.L. wrote the paper.

Conflicts of Interest: The authors declare no conflict of interest.

\section{Appendix A}

Simplifying Equation (13), it is expressed as (take $k_{2}>0$ as an example):

$$
\phi_{\text {ion }}(\mathbf{P})=\arg \left\{\exp \left[-j \frac{4 \pi K}{c f_{0}}\left(T E C_{0}(\mathbf{P})-\frac{k_{1}(\mathbf{P})^{2}}{4 k_{2}(\mathbf{P})}\right)\right] \cdot \int_{-\frac{T_{a} \sqrt{k_{2}(\mathbf{P})}}{2}+\frac{k_{1}(\mathbf{P})}{2 \sqrt{k_{2}(\mathbf{P})}}}^{\frac{T_{a} \sqrt{k_{2}(\mathbf{P})}}{2}+\frac{k_{1}(\mathbf{P})}{2 \sqrt{k_{2}(\mathbf{P})}}} \exp \left[-j \frac{4 \pi K}{c f_{0}} \cdot u^{2}\right] d u\right\}
$$

Using $u=\sqrt{k_{2}(\mathbf{P})} t_{a}+\frac{k_{1}(\mathbf{P})}{2 \sqrt{k_{2}(\mathbf{P})}}$ and $y=\sqrt{\frac{8 K}{c f_{0}}} u$ to realize the variable substitution in Equation (10) and expanding it by Euler's formula, we have:

$$
\phi_{i o n}(\mathbf{P})=\arg \left\{\exp \left[-j \frac{4 \pi K}{c f_{0}}\left(T E C_{0}(\mathbf{P})-\frac{k_{1}(\mathbf{P})^{2}}{4 k_{2}(\mathbf{P})}\right)\right] \cdot \sqrt{\frac{c f_{0}}{8 K^{2}}} \int_{\varepsilon_{2}}^{\varepsilon_{1}}\left[\cos \left(\frac{\pi}{2} y^{2}\right)-j \sin \left(\frac{\pi}{2} y^{2}\right)\right] d y\right\}
$$

where

$$
\left\{\begin{aligned}
\varepsilon_{1} & =\sqrt{\frac{8 K}{c f_{0}}}\left(\frac{T_{a} \sqrt{k_{2}(\mathbf{P})}}{2}+\frac{k_{1}(\mathbf{P})}{2 \sqrt{k_{2}(\mathbf{P})}}\right) \\
\varepsilon_{2} & =\sqrt{\frac{8 K}{c f_{0}}}\left(-\frac{T_{a} \sqrt{k_{2}(\mathbf{P})}}{2}+\frac{k_{1}(\mathbf{P})}{2 \sqrt{k_{2}(\mathbf{P})}}\right)
\end{aligned}\right.
$$

Since there are two Fresnel integrals inside Equation (A2), we apply the series expansions to them. Then, we obtain:

$$
\phi_{\text {ion }}(\mathbf{P})=\frac{4 \pi K}{c f_{0}}\left(T E C_{0}(\mathbf{P})-\frac{k_{1}(\mathbf{P})^{2}}{4 k_{2}(\mathbf{P})}+\Phi_{h}\left(k_{1}(\mathbf{P}), k_{2}(\mathbf{P})\right)\right)
$$

where $\Phi_{h}\left(k_{1}(\mathbf{P}), k_{2}(\mathbf{P})\right)$ is expressed as

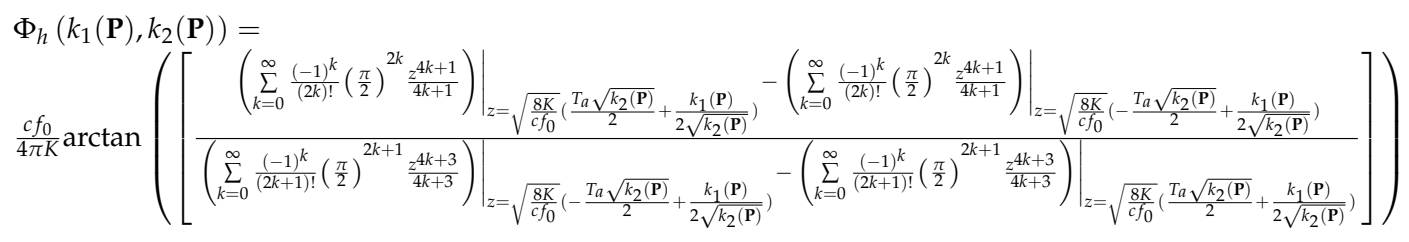

where $z$ and $k$ are the variables related to the series.

Especially when (A3) is small, we have approximately:

$$
\phi_{\text {ion }}(\mathbf{P})=\frac{4 \pi K}{c f_{0}}\left(T E C_{0}(\mathbf{P})-\frac{k_{1}(\mathbf{P})^{2}}{4 k_{2}(\mathbf{P})}+\frac{c f_{0}}{4 \pi K} \arctan \left[\frac{4 \pi K}{3 c f_{0}}\left(\frac{3 k_{1}(\mathbf{P})^{2}}{4 k_{2}(\mathbf{P})}+\frac{T_{a}^{2} k_{2}(\mathbf{P})}{4}\right)\right]\right)
$$




\section{References}

1. Dong, X.C.; Hu, C.; Tian, Y.; Tian, W.M.; Li, Y.H.; Long, T. Experimental study of ionospheric impacts on geosynchronous SAR using GPS signals. IEEE J. Sel. Top. Appl. Earth Obs. Remote Sens. 2016, 9, 2171-2183. [CrossRef]

2. Bruno, D.; Hobbs, S.E.; Ottavianelli, G. Geosynchronous synthetic aperture radar: Concept design, properties and possible applications. Acta Astronaut. 2006, 59, 149-156. [CrossRef]

3. Tomiyasu, K. Synthetic aperture radar in geosynchronous orbit. In Proceedings of the Digest International IEEE Antennas Propagation Symposium, College Park, MD, USA, 15-19 March 1978.

4. Hobbs, S.; Mitchell, C.; Forte, B.; Holley, R.; Snapir, B.; Whittaker, P. System design for geosynchronous synthetic aperture radar missions. IEEE Trans. Geosci. Remote Sens. 2014, 52, 7750-7763. [CrossRef]

5. Prati, C.; Rocca, F.; Giancola, D.; MontiGuarnieri, A. Passive geosynchronous SAR system reusing backscattered digital audio broadcasting signals. IEEE Trans. Geosci. Remote Sens. 1998, 36, $1973-1976$. [CrossRef]

6. Qi, L.; Tan, W.; Lin, Y.; Wang, Y.; Hong, W.; Wu, Y. SAR raw data 2-D imaging model and simulation of GEO CSAR. In Proceedings of the IEEE CIE International Conference Radar, Chengdu, China, 24-27 October 2011.

7. Hu, B.; Jiang, Y.; Zhang, S.; Zhang, Y.; Yeo, T. Generalized omega-K algorithm for geosynchronous SAR image formation. IEEE Geosci. Remote Sens. Lett. 2015, 12, 2286-2290. [CrossRef]

8. Madsen, S.N.; Edelstein, W.; Di domenico, L.; Labreque, J. A geosynchronous synthetic aperture radar for tectonic mapping, disaster management and measurement of vegetation and soil moisture. In Proceedings of the IEEE IGARSS, Sydney, Australia, 9-13 July 2001.

9. Massonnet, D.; Rossi, M.; Carmona, C.; Ardagna, F.; Peltzer, G.; Feigl, K.; Rabaute, T. The displacement field of the landers earthquake mapped by radar interferometry. Nature 1993, 364, 138-142. [CrossRef]

10. Mantovani, M.; Devoto, S.; Piacentini, D.; Prampolini, M.; Soldati, M.; Pasuto, A. Advanced SAR interferometric analysis to support geomorphological interpretation of slow-moving coastal landslides (Malta, Mediterranean Sea). Remote Sens. 2016, 8, 443. [CrossRef]

11. Ferretti, A.; MontiGuarnieri, A.; Prati, C.; Rocca, F.; Massonnet, D. InSAR Principles: Guideline for SAR Interferometry Processing and Interpretation; ESA Publications: Noordwijk, The Netherlands, 2007; pp. 57-71.

12. Hanssen, R.F. Radar Interferometry: Data Interpretation and Error Analysis; Kluwer Academic Publishers: New York, NY, USA, 2001; pp. 23-60.

13. MontiGuarnieri, A.; Prati, C. ScanSAR focusing and interferometry. IEEE Trans. Geosci. Remote Sens. 1996, 34, 1029-1038.

14. Qu, F.; Lu, Z.; Poland, M.; Freymueller, J.; Zhang, Q.; Jung, H.S. Post-eruptive inflation of okmok volcano, from InSAR, 2008-2014. Remote Sens. 2015, 7, 16778-16794. [CrossRef]

15. Li, Y.H.; Hu, C.; Long, T. A novel SAR interferometry processing method in high resolution spotlight SAR. J. Electromagn. Waves Appl. 2015, 29, 1786-1803. [CrossRef]

16. Madsen, S.N.; Chen, C.; Edelstein, W. Radar options for global earthquake monitoring. In Proceedings of the IGARSS, Toronto, ON, Canada, 24-28 June 2002.

17. MontiGuarnieri, A.; Broquetas, A.; Recchia, A.; Rocca, F.; Ruiz-Rodon, J. Advanced radar geosynchronous observation system: ARGOS. IEEE Geosci. Remote Sens. Lett. 2015, 12, 1406-1410. [CrossRef]

18. Recchia, A.; MontiGuarnieri, A.; Broquetas, A.; Leanza, A. Impact of scene decorrelation on geosynchronous SAR data focusing. IEEE Trans. Geosci. Remote Sens. 2015, 54, 1635-1646. [CrossRef]

19. Hu, C.; Li, Y.H.; Dong, X.C.; Long, T. Optimal data acquisition and height retrieval in repeat-track geosynchronous SAR interferometry. Remote Sens. 2015, 7, 13367-13389. [CrossRef]

20. Xu, Z.W.; Wu, J.; Wu, Z.S. A survey of ionosphere effects on space-based radar. Waves Random Media 2004, 14, 189-273. [CrossRef]

21. Yeh, K.C.; Liu, C.H. Radio wave scintillations in the ionosphere. Proc. IEEE 1982, 70, 324-360.

22. Ishimaru, A.; Kuga, Y.; Liu, J.; Kim, Y.; Freeman, T. Ionospheric effects on synthetic aperture radar at $100 \mathrm{MHz}$ to $2 \mathrm{GHz}$. Radio Sci. 1999, 7, 257-268. [CrossRef]

23. Meyer, F.J. Performance requirements for ionospheric correction of low-frequency SAR data. IEEE Trans. Geosci. Remote Sens. 2011, 49, 3694-3702. [CrossRef]

24. Jehle, M.; Frey, O.; Small, D.; Meier, E. Measurement of ionospheric TEC in spaceborne SAR data. IEEE Trans. Geosci. Remote Sens. 2010, 48, 2460-2468. [CrossRef] 
25. Tian, Y.; Hu, C.; Dong, X.C.; Zeng, T.; Long, T.; Lin, K.; Zhang, X.Y. Theoretical analysis and verification of time variation of background ionosphere on geosynchronous SAR imaging. IEEE Geosci. Remote Sens. Lett. 2015, 12, 721-725. [CrossRef]

26. Li, L.; Hong, J. Study of ionospheric effects on azimuth imaging for medium-earth-orbit SAR. Prog. Electromagn. Res. 2015, 32, 215-228. [CrossRef]

27. Li, Y.H.; Hu, C.; Dong, X.C.; Tian, W.M.; Long, T. Impacts of ionospheric scintillation on geosynchronous SAR focusing: Preliminary experiments and analysis. Sci. China Inf. Sci. 2015, 58, 109301-109303. [CrossRef]

28. Wang, C.; Zhang, M.; Xu, Z.W.; Chen, C.; Sheng, D.S. Effects of anisotropic ionospheric irregularities on space-borne SAR imaging. IEEE Trans. Antennas Propag. 2014, 62, 4664-4673. [CrossRef]

29. Meyer, F.; Chotoo, K.; Chotoo, S.; Huxtable, B.; Carrano, C. The influence of equatorial scintillation on L-band SAR image quality and phase. IEEE Trans. Geosci. Remote Sens. 2016, 55, 869-880. [CrossRef]

30. Zeng, T.; Wang, R.; Li, F. SAR image autofocus utilizing minimum-entropy criterion. IEEE Geosci. Remote Sens. Lett. 2013, 10, 1552-1556. [CrossRef]

31. Hu, C.; Li, Y.H.; Dong, X.C.; Ao, D.Y. Avoiding the ionospheric scintillation interference on geosynchronous SAR by orbit optimization. IEEE Geosci. Remote Sens. Lett. 2016, 13, 1676-1680. [CrossRef]

32. U.S. Total Electron Content. Available online: www.swpc.noaa.gov/products/us-total-electron-content (accessed on 2 November 2016).

33. Ferretti, A.; Prati, C.; Rocca, F. Permanent scatterers in SAR interferometry. IEEE Trans. Geosci. Remote Sens. 2001, 39, 8-20. [CrossRef]

34. Hilley, G.; Bürgmann, R.; Ferretti, A.; Novali, F.; Rocca, F. Dynamics of slow-moving landslides from permanent scatterer analysis. Science 2004, 304, 1952-1955. [CrossRef] [PubMed]

35. Tofani, V.; Raspini, F.; Catani, F.; Casagli, N. Persistent Scatterer Interferometry (PSI) technique for landslide characterization and monitoring. Remote Sens. 2013, 5, 1045-1065. [CrossRef]

36. Vajedian, S.; Motagh, M.; Nilfouroushan, F. StaMPS improvement for deformation analysis in mountainous regions for the Damavand Volcano and Mosha Fault in Alborz. Remote Sens. 2015, 7, 8323-8347. [CrossRef]

37. Jung, H.S.; Won, J.S.; Kim, S.W. An improvement of the performance of multiple-aperture SAR interferometry (MAI). IEEE Trans. Geosci. Remote Sens. 2009, 47, 2859-2869. [CrossRef]

(C) 2016 by the authors; licensee MDPI, Basel, Switzerland. This article is an open access article distributed under the terms and conditions of the Creative Commons Attribution (CC-BY) license (http://creativecommons.org/licenses/by/4.0/). 